\title{
All-optical Label Extractor/Eraser for In-band Labels and 160 Gb/s Payload Based on Micro Ring Resonators
}

\author{
N. Calabretta, P.J. Urban, *D.H. Geuzebroek, **E.J. Klein, H. de Waardt, and H J.S. Dorren \\ COBRA Research Institute, Eindhoven University of Technology, PO. Box 512, 5600MB - Eindhoven, The Netherlands \\ *Lionix BV, PO Box 456, 7500 AH-Enschede, The Netherlands, **XiO Photonics, PO Box 1254, 7500 BG, Enschede, The Netherlands \\ n.calabretta@tue.nl
}

\begin{abstract}
We demonstrate a passive and asynchronous photonic integrated all-optical label extractor/eraser by using narrow-band silicon-nitride add/drop ring-resonators. Error-free simultaneous separation/erasing of the in-band labels from the $160 \mathrm{~Gb} / \mathrm{s}$ packet payload was demonstrated without pulse-shape distortions.
\end{abstract}

(C) 2009 Optical Society of America

OCIS codes: (060.6719) Switching, packet; (200.4740) Optical processing; (130.7408) Wavelength filtering devices.

\section{Introduction}

All-optical packet switching based on all-optical signal processing is a promising technology to solve the mismatch between the fibre bandwidth and the router forwarding capacity [1-2]. Moreover, it has been argued that all-optical technology can profit from the capability to integrate all the required switching functionalities in a photonic integrated chip to allow a great reduction of volume, power consumption and costs. It is then an essential issue to provide all-optical signal processing functionalities that are suitable for photonic integration.

Recently an all-optical label extractor/eraser for in-band labeling addresses and $160 \mathrm{~Gb} / \mathrm{s}$ data payload was successfully employed to demonstrate a 1x4 all-optical packet switch (AOPS) [3]. The AOPS schematic and the packet format are shown in fig. 1. The optical address is encoded by combining the in-band labels (the wavelengths of the labels are located within the $20 \mathrm{~dB}$ bandwidth of the payload). In-band labelling technique has the advantage that label extraction can be implemented by using passive optical band-pass filters centered at the labels wavelength and label erasing by using notch filters. In [3], the label extractor/eraser was implemented by exploiting the reflection and pass-through ports of two pigtailed fiber Bragg gratings (FBG) centered at the labels wavelength and two optical circulator (see fig. 1). Thus, the labels, reflected by the FBGs, are extracted via the optical circulators, while the data payload passes through the FBGs and is fed into the switching fabric. The extracted labels are optically processed by the label processor, which produces an optical routing signal driving the switching fabric.

Despite the successful demonstration of the AOPS and the potential integration of the label processor and switching fabric [3], photonic integration of the entire all-optical packet switch is prevented by the integration of the label extractor/eraser. Moreover, the broad bandwidth of the FBGs caused a splicing and distortion of the spectrum of the payload which resulted in a pulse broadening of the $160 \mathrm{~Gb} / \mathrm{s}$ payload. This leads to a closed eye and therefore to an extra power penalty. The pulse distortion can be practically avoided by using optical band-pass filters with narrow bandwidth and optical notch filters with narrow stop-band and flat all-pass-band.

In this work, we demonstrate a label extractor/eraser for in-band labeling addresses and $160 \mathrm{~Gb} / \mathrm{s}$ payload by using an integrated micro-ring resonator with pass-trough and drop ports. By exploiting the narrow bandwidth of the drop port and the narrow bandwidth and flat all-pass band of the pass-through port simultaneous error-free label extraction and label erasing has been performed without noticeable pulse distortion.

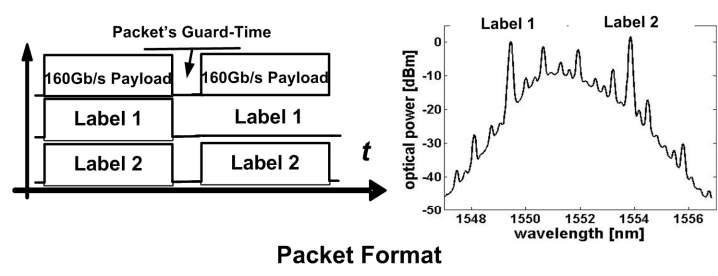

a)

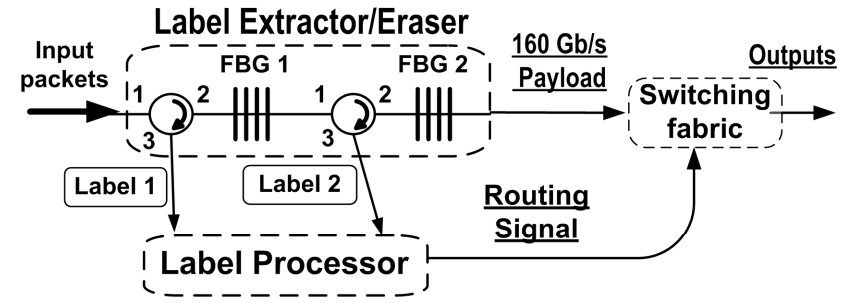

b)

Figure 1. a) Format of the packets illustrating the in-band labelling technique. b) All-optical packet switch configuration. 


\section{Experiments and Results}

The experimental set-up employed to demonstrate the micro-ring based label extractor/eraser is shown in Fig. 2. The packet format is the same as the one shown in Fig. 1. Packet payload is generated by time-quadrupling a $40 \mathrm{~Gb} / \mathrm{s}$ data-stream consisting of 256 pre-defined return-to-zero bits $\left(\lambda_{\mathrm{p}}=1551.6 \mathrm{~nm}\right)$ into a $160 \mathrm{~Gb} / \mathrm{s}$ data-stream using a passive pulse interleaver. Each pulse has duration of $1.5 \mathrm{ps}$ making the $20 \mathrm{~dB}$ bandwidth of the payload to be $5 \mathrm{~nm}$. The resulting packet payload consists of a $5.6 \mathrm{~ns}$ data burst. The guard-time between the packets is 800 ps. We employ in-band labelling; the packet address information is encoded with $\mathrm{CW}$-signals at wavelengths within the 5 nm payload bandwidth. Each label can have a binary value: ' 0 ' means no signal at the label wavelength, ' 1 ' means an optical signal at the label wavelength. The duration of the label equals the duration of the payload. We encode the addresses by using two in-band labels at $\lambda_{L I}=1549.45 \mathrm{~nm}$ and $\lambda_{L 2}=1553.85 \mathrm{~nm}$. Note that the two CW-signals are modulated by one single amplitude modulator driven by a low speed pattern generator.

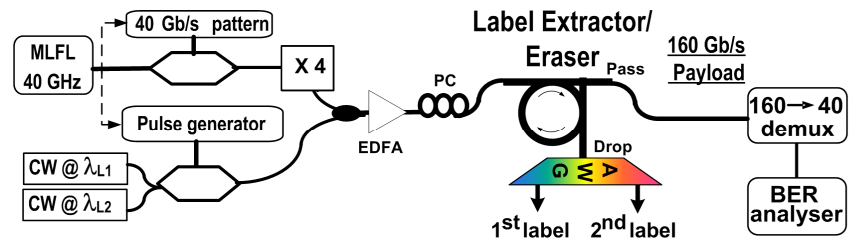

a)

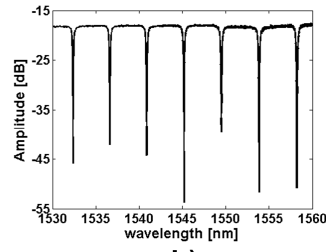

b)

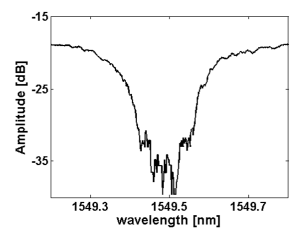

c)

Fig. 2. a) Experimental set-up; b) transfer function of the pass-through; c) A magnification of one of the stop band.

The label extractor/eraser consists of a pigtailed vertically coupled micro-ring resonators fabricated in the $\mathrm{Si} 3 \mathrm{~N} 4 / \mathrm{SiO} 2$ materials system [4] (high contrast materials system, $\Delta \mathrm{n} \approx 0.55$ ), with a pass-through port and a drop port as shown in Fig. 2a. The transfer function of the pass-through port is reported in Fig. 2b. The FSR of the periodic stop-bands is $4.4 \mathrm{~nm}$. A magnification of one of the stop band is shown in Fig. $2 \mathrm{c}$. The measured $-3 \mathrm{~dB}$ bandwidth was $0.16 \mathrm{~nm}$ and the flatness of the pass-band was $+/-0.4 \mathrm{~dB}$. The total loss of the device is $20 \mathrm{~dB}$, of which $16 \mathrm{~dB}$ are coupling losses and $4 \mathrm{~dB}$ are waveguide losses in the ring.

The optical packets are amplified and processed by the label extractor/eraser. A polarization controller controls the input polarization to the micro-ring. The stop-bands at multiple FSR are designed to be at the wavelengths matching the labels wavelengths. Therefore, the labels wavelengths are filter out at the drop-port, while the wavelength payload is output to the pass-through port. The two labels at wavelength $\lambda_{\mathrm{L} 1}$ and $\lambda_{\mathrm{L} 2}$ are output from the drop port and then separated by an AWG filter ready to be fed into the label processor. This is confirmed by spectral and time domain measurements. In Fig. 3 the optical spectra measurements with resolution bandwidth of $0.06 \mathrm{~nm}$ at the drop and pass-through ports are reported. The optical spectrum of the input packet before the label extractor is shown in Fig. 3a. The optical spectrum at the output of the drop port and the pass-through output port are reported in Fig. 3bc. More than $25 \mathrm{~dB}$ of separation/erasing between the labels and the optical payload was measured.
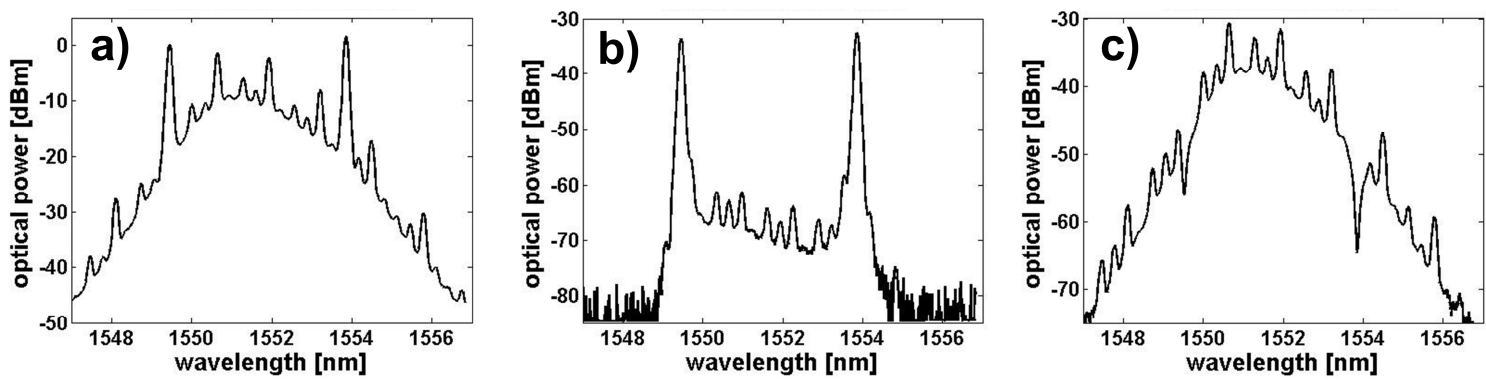

Figure 3. Measured optical spectra. a) Optical packets at the input of the label extractor; b) Output of the drop port showing the optical spectra of the two labels; c) Output of the pass-through port showing the optical spectrum of the $160 \mathrm{~Gb} / \mathrm{s}$ payload .

We also report in Fig. 4a-c the time-domain traces of the $160 \mathrm{~Gb} / \mathrm{s}$ payload and the extracted labels traces (after the AWG) recorded at the pass-trough port and drop port, respectively. Note that the two labels have the same pattern, because we employed a single amplitude modulator driven by a pattern generator to modulate the two CW-signals at $\lambda_{\mathrm{L} 1}$ and $\lambda_{\mathrm{L} 2}$. The labels present no shape distortion and no visible cross-talk due to the payload.

The eye diagrams of the original input $160 \mathrm{~Gb} / \mathrm{s}$ data payload and of the $160 \mathrm{~Gb} / \mathrm{s}$ payload after the label extractor/eraser are reported in Fig. 4d-e. An optical sample oscilloscope with a $700 \mathrm{GHz}$ of bandwidth was employed to record the eye diagrams of the $160 \mathrm{~Gb} / \mathrm{s}$ pulses. Very small degradation and broadening of the pulses is 
observed after the label extraction. The measured pulse-width broadening was 0.4 ps. The measured RMS time jitter was $212 \mathrm{fs}$ for the pulses after the label extractor/eraser, which results in an increase of $40 \mathrm{fs}$ compared to the $173 \mathrm{fs}$ of the input $160 \mathrm{~Gb} / \mathrm{s}$ payload pulses. For comparison, we also reported in Fig. $4 \mathrm{f}$ the eye diagram of the 160 $\mathrm{Gb} / \mathrm{s}$ payload after the label extraction implemented by using the FBGs as in [3]. A much larger pulse broadening can be observed caused by the broad bandwidth of the FBGs $(-3 \mathrm{~dB}$ bandwidth of $0.42 \mathrm{~nm}$ and $0.1 \mathrm{~nm}$ for FBG1 and FBG 2, respectively) compared to the bandwidth of the micro-ring add/drop resonator.

To quantify the performance of the label extractor/eraser, BER measurements are reported in Fig. 4. The BER measurements were performed in a static operation by time-quadrupling $40 \mathrm{~Gb} / \mathrm{s}$ PRBS $2^{31}-1$ data payload. The resulting $160 \mathrm{~Gb} / \mathrm{s}$ data payload after the label extractor/eraser is amplified and time-demultiplexed from $160 \mathrm{~Gb} / \mathrm{s}$ to $40 \mathrm{~Gb} / \mathrm{s}$ by using an electrically clocked EAM that creates a 5 ps switching window with a periodicity of 25 ps. The resulting $40 \mathrm{~Gb} / \mathrm{s}$ data is then detected by a $40 \mathrm{~Gb} / \mathrm{s}$ detector and analyzed by using a BER tester. As reference we report the BER curve of the back-to-back (b-t-b) $160 \mathrm{~Gb} / \mathrm{s}$ payload. The BER curve of the $160 \mathrm{~Gb} / \mathrm{s}$ payload after the label extraction/eraser shows error-free operation with limited power penalty of around $0.5 \mathrm{~dB}$. The power penalty can be ascribed to the additional ASE noise introduced by the amplification stage required to compensate the total loss of the label extractor/extractor.

a)

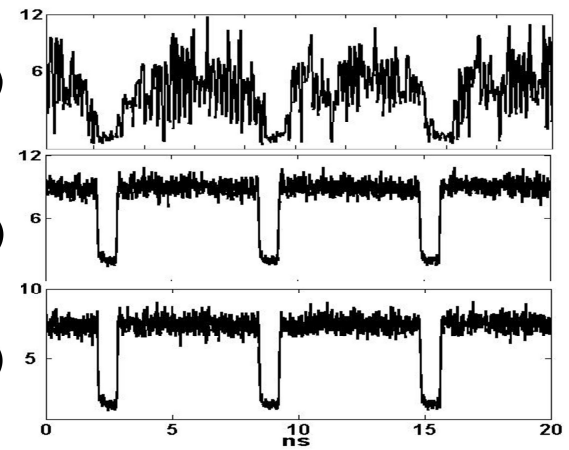

d)

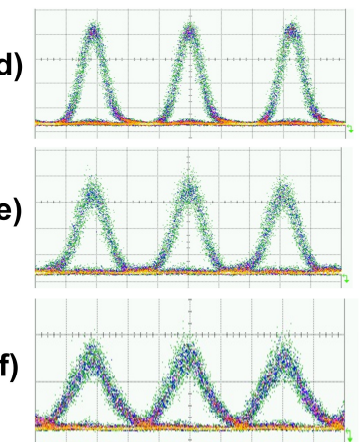

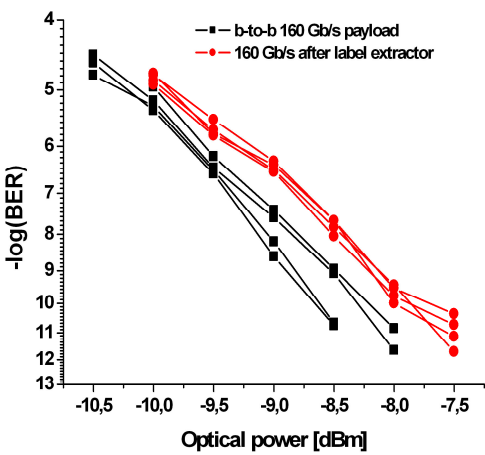

Figure 4. a) $160 \mathrm{~Gb} / \mathrm{s}$ payload after the label extractor; b-c) Extracted Label 1 and Label 2. The vertical scale is mV/div. d) Eye diagram of the input $160 \mathrm{~Gb} / \mathrm{s}$ payload; e) Eye diagram of the $160 \mathrm{~Gb} / \mathrm{s}$ payload after the label extractor/eraser; f) Eye diagram of the $160 \mathrm{~Gb} / \mathrm{s}$ payload after the label extractor/eraser employed in Ref.[3]. The time scale is 2 ps/div. g) BER measurements before and after the label extractor/eraser.

\section{Conclusions}

We have demonstrated error-free operation of a photonic integrated all-optical label extractor/eraser for in-band labelling addresses by using a passive silicon-nitride micro-ring add/drop resonator. The in-band labels and the 160 $\mathrm{Gb} / \mathrm{s}$ payload of the optical packets were successfully separated to the drop port and pass-through port, respectively. Due to the narrow stop-band and flatness of the pass-band of the pass-through port of the micro-ring, almost no distortion is experienced by $160 \mathrm{~Gb} / \mathrm{s}$ payload spectrum. As a result, the $160 \mathrm{~Gb} / \mathrm{s}$ payload pulses exhibit very limited broadening, in contrast with the visible broadening experienced by the payload pulses by using FBGs with broader stop-band [3]. This resulted in a low power penalty of $0.5 \mathrm{~dB}$. The negligible spectral distortion of the payload due to the utilization of micro-ring with a narrow stop-band is also very important in the perspective to operate the label extractor/eraser with a larger number of labels. We are currently designing for the realization of a micro-ring resonator capable of extracting/erasing a larger number of in-band labels. Moreover, novel design will benefit from the achievement in technology to realize polarization independent and low insertion loss micro-ring add/drop resonator as demonstrated in [5-6]. Finally, it is worth to note that since the previous implementation of the label extractor/eraser employed in [3] was not suitable for photonic integration, the photonic integration of the alloptical packet switch node was prevented. This work demonstrates the feasibility for photonic integration of the label extractor/eraser, which may allow for photonic integration of the entire all-optical packet switch configuration. This work was supported by the Dutch Government under the Freeband BB Photonics project BSIK 03025.

\section{References}

[1] D. J. Blumenthal et al., "All-optical label swapping networks and technologies", JLT 18, 2058 (2000).

[2] J. Herrera et al., "160 Gb/s all-optical packet switched network operation over 110km of field installed fiber", Proc. OFC2007, PDP4(2007).

[3] N. Calabretta et. al., '1 x 4 all-optical packet switch at $160 \mathrm{~Gb} / \mathrm{s}$ employing optical processing of scalable in-band address labels,' OFC 2008, San Diego, CA, PDP 33 (2008).

[4] E. J. Klein et al., 'Densely Integrated Microring Resonator based Photonic Devices for use in Access Networks,' Optics Express, 15, 1034610355 (2007).

[5] F. Morichetti et al., 'Box-Shaped dielectric waveguides: a new concept in integrated optics?,' JLT 25, 2579-2589 (2007).

[6] www.xiophotonics.com 